\title{
SPACES OF ABSOLUTELY SUMMING POLYNOMIALS
}

\author{
J. A. BARBOSA, G. BOTELHO, D. DINIZ and D. PELLEGRINO*
}

\begin{abstract}
This paper has a twofold purpose: to prove a much more general Dvoretzky-Rogers type theorem for absolutely summing polynomials and to introduce a more convenient norm on the space of everywhere summing polynomials.
\end{abstract}

\section{Introduction}

Since Pietsch [16], several nonlinear generalizations of absolutely summing operators have been investigated. Multilinear mappings/polynomials which are absolutely summing at a given point - and also everywhere - were introduced by M. Matos [9] and developed in [3], [7], [13], [14].

It is known that a Dvoretzky-Rogers-like theorem holds for everywhere summing polynomials (see [9]) but does not hold for summing polynomials (at the origin), so it is natural to ask whether or not such a theorem holds for polynomials which are absolutely summing at a point $a \neq 0$. Proving in Section 3 a Dvoretzky-Rogers type theorem for absolutely summing polynomials at a given point $a \neq 0$, we provide a substantial improvement of Matos' Dvoretzky-Rogers type theorem [9]. We also prove that summability at any point implies summability at the origin.

The norm that has been used in the space of everywhere summing polynomials (defined in [9]) has two inconvenients: (i) it is not a normalized ideal norm, in the sense that the everywhere summing norm of the polynomial $x \longrightarrow x^{n}, x \in \mathrm{K}=$ scalar field, is not always equal to 1; (ii) it makes computations quite difficult. In Section 4 we introduce another norm which happens to be equivalent to the original one and repairs the aforementioned inconvenients. The multilinear case is also investigated.

\section{Background and notation}

Recall that, if $E$ and $F$ are Banach spaces over $\mathrm{K}=\mathrm{R}$ or $\mathrm{C}$ and $p \geq q \geq 1$, a continuous linear operator $u: E \longrightarrow F$ is absolutely $(p ; q)$-summing

\footnotetext{
* The second and fourth named authors are partially supported by IM-AGIMB. The fourth named author is also supported by $\mathrm{CNPq} /$ Fapesq.

Received March 14, 2006.
} 
(or $(p ; q)$-summing) if $\left(u\left(x_{j}\right)\right)_{j=1}^{\infty}$ is absolutely $p$-summable in $F$ whenever $\left(x_{j}\right)_{j=1}^{\infty}$ is weakly $q$-summable in $E$. For the theory of absolutely summing operators we refer to the book by Diestel-Jarchow-Tonge [4].

The multilinear theory of absolutely summing operators was introduced by Pietsch [16] and has been developed by several authors. There are various natural possible generalizations of the linear concept of absolute summability to polynomial/multilinear mappings (see [1], [5], [7], [10], [15]). If $u$ is a linear operator, to estimate $\left(u\left(a+x_{j}\right)-u(a)\right)_{j=1}^{\infty}$ is the same as to estimate $\left(u\left(x_{j}\right)\right)_{j=1}^{\infty}$. However, for polynomials, in general, $P(a+x) \neq P(a)+P(x)$, as well as for multilinear mappings and hence, in the nonlinear case it makes sense to study absolute summabilitily with respect to a point $a \neq 0$. This idea is credited to Richard Aron, appeared for the first time in M. Matos [8] and was developed in [9] and in the doctoral thesis [12] of the fourth named author under supervision of Professor M. Matos.

As usual, the Banach space of all continuous $n$-homogeneous polynomials from $E$ into $F$, with the sup norm, is represented by $\mathscr{P}\left({ }^{n} E ; F\right)$. The sequence spaces $\ell_{p}(E)$ and $\ell_{p}^{u}(E)$ are defined by:

$$
\begin{aligned}
\ell_{p}(E)=\left\{\left(x_{j}\right)_{j=1}^{\infty} \in E^{\mathrm{N}} ;\left\|\left(x_{j}\right)_{j=1}^{\infty}\right\|_{p}:=\left(\sum_{j=1}^{\infty}\left\|x_{j}\right\|^{p}\right)^{\frac{1}{p}}<\infty\right\}, \\
\ell_{p}^{w}(E)=\left\{\left(x_{j}\right)_{j=1}^{\infty} \in E^{\mathrm{N}} ;\left\|\left(x_{j}\right)_{j=1}^{\infty}\right\|_{w, p}:=\sup _{\varphi \in B_{E^{\prime}}}\left(\sum_{j=1}^{\infty}\left|\varphi\left(x_{j}\right)\right|^{p}\right)^{\frac{1}{p}}<\infty\right. \\
\text { and } \left.\lim _{k \rightarrow \infty}\left\|\left(x_{j}\right)_{j=k}^{\infty}\right\|_{w, p}=0\right\} .
\end{aligned}
$$

A polynomial $P \in \mathscr{P}\left({ }^{n} E ; F\right)$ is $(p ; q)$-summing at $a \in E$ if $\left(P\left(a+x_{j}\right)-\right.$ $P(a))_{j=1}^{\infty} \in \ell_{p}(F)$ for every $\left(x_{j}\right)_{j=1}^{\infty} \in \ell_{q}^{u}(E)$. It is not hard to prove that the class of all $n$-homogeneous polynomials from $E$ into $F$ that are absolutely summing at a given point is a subspace of $\mathscr{P}\left({ }^{n} E ; F\right)$. The space formed by the $n$-homogeneous polynomials that are ( $p ; q)$ summing at $a \in E$ will be denoted by $\mathscr{P}_{a s(p ; q)}^{(a)}\left({ }^{n} E ; F\right)$. The $n$-homogeneous polynomials that are $(p ; q)$-summing at $a=0$ will be simply called $(p ; q)$-summing and the vector space of all $(p ; q)$-summing $n$-homogeneous polynomials from $E$ into $F$ is represented by $\mathscr{P}_{a s(p ; q)}\left({ }^{n} E ; F\right)$.

The space composed by the $n$-homogeneous polynomials that are $(p ; q)$ summing at every point is denoted by $\mathscr{P}_{a s(p ; q)}^{e v}\left({ }^{n} E ; F\right)$. Note that

$$
\mathscr{P}_{a s(p ; q)}^{e v}\left({ }^{n} E ; F\right)=\bigcap_{a \in E} \mathscr{P}_{a s(p ; q)}^{(a)}\left({ }^{n} E ; F\right) .
$$


If $P \in \mathscr{P}_{a s(p ; q)}^{e v}\left({ }^{n} E ; F\right)$ we say that $P$ is everywhere $(p ; q)$-summing. The space of all continuous $n$-linear mappings from $E_{1} \times \cdots \times E_{n}$ into $F$ (with the sup norm) is denoted by $\mathscr{L}\left(E_{1}, \ldots, E_{n} ; F\right)\left(\mathscr{L}\left({ }^{n} E ; F\right)\right.$ if $E_{1}=\cdots=$ $\left.E_{n}=E\right)$. We say that $T \in \mathscr{L}\left(E_{1}, \ldots, E_{n} ; F\right)$ is $\left(p ; q_{1}, \ldots, q_{n}\right)$-summing at $a=\left(a_{1}, \ldots, a_{n}\right) \in E_{1} \times \cdots \times E_{n}$ if

$$
\left(T\left(a_{1}+x_{j}^{(1)}, \ldots, a_{n}+x_{j}^{(n)}\right)-T\left(a_{1}, \ldots, a_{n}\right)\right)_{j=1}^{\infty} \in \ell_{p}(F)
$$

for every $\left(x_{j}^{(r)}\right)_{j=1}^{\infty} \in \ell_{q_{r}}^{u}\left(E_{r}\right), r=1, \ldots, n$. As it happens for polynomials, it is easy to verify that the class of all $n$-linear mappings from $E_{1} \times$ $\cdots \times E_{n}$ into $F$ which are $\left(p ; q_{1}, \ldots, q_{n}\right)$-summing at $a$, represented by $\mathscr{L}_{a s\left(p ; q_{1}, \ldots, q_{n}\right)}^{(a)}\left(E_{1}, \ldots, E_{n} ; F\right)$, is a subspace of $\mathscr{L}\left(E_{1}, \ldots, E_{n} ; F\right)$. The space formed by the $n$-linear mappings from $E_{1} \times \cdots \times E_{n}$ into $F$ which are $\left(p ; q_{1}, \ldots, q_{n}\right)$-summing at every point is denoted by $\mathscr{L}_{a s\left(p ; q_{1}, \ldots, q_{n}\right)}\left(E_{1}, \ldots\right.$, $\left.E_{n} ; F\right)$. If $T \in \mathscr{L}_{a s\left(p ; q_{1}, \ldots, q_{n}\right)}^{e v}\left(E_{1}, \ldots, E_{n} ; F\right)$ we say that $T$ is everywhere $\left(p ; q_{1}, \ldots, q_{n}\right)$-summing. The $n$-linear mappings that are $\left(p ; q_{1}, \ldots, q_{n}\right)$-summing at $a=0$ will be simply called $\left(p ; q_{1}, \ldots, q_{n}\right)$-summing and the vector space of all $\left(p ; q_{1}, \ldots, q_{n}\right)$-summing $n$-linear mappings from $E_{1} \times \cdots \times E_{n}$ into $F$ is represented by $\mathscr{L}_{a s\left(p ; q_{1}, \ldots, q_{n}\right)}\left(E_{1}, \ldots, E_{n} ; F\right)$.

If $p=q=q_{1}=\cdots=q_{n}$, instead of $(p ; p)$ or $(p ; p, \ldots, p)$-summing we say that the mapping is $p$-summing. In this case we write $\mathscr{P}_{a s, p}^{(a)}\left({ }^{n} E ; F\right)$, $\mathscr{P}_{a s, p}\left({ }^{n} E ; F\right)$ and $\mathscr{P}_{a s, p}^{e v}\left({ }^{n} E ; F\right)$ for polynomials, and the adaptations for multilinear mappings are obvious.

Nachbin's concept of holomorphy type [11] was generalized in a natural way in [3] in the following fashion: a global holomorphy type $\mathscr{P}_{H}$ is a subclass of the class of all continuous homogeneous polynomials between Banach spaces such that for every natural $n$ and every Banach spaces $E$ and $F$, the component $\mathscr{P}_{H}\left({ }^{n} E ; F\right):=\mathscr{P}\left({ }^{n} E ; F\right) \cap \mathscr{P}_{H}$ is a linear subspace of $\mathscr{P}\left({ }^{n} E ; F\right)$ which is a Banach space when endowed with a norm denoted by $P \rightarrow\|P\|_{H}$, and

(i) $\mathscr{P}_{H}\left({ }^{0} E ; F\right)=F$, as a normed linear space for all $E$ and $F$.

(ii) There is $\sigma \geq 1$ such that for every Banach spaces $E$ and $F, n \in \mathrm{N}$, $k \leq n, a \in E$ and $P \in \mathscr{P}_{H}\left({ }^{n} E ; F\right), \hat{d}^{k} P(a) \in \mathscr{P}_{H}\left({ }^{k} E ; F\right)$ and

$$
\left\|\frac{1}{k !} \hat{d}^{k} P(a)\right\|_{H} \leq \sigma^{n}\|P\|_{H}\|a\|^{n-k},
$$

where $\hat{d}^{k} P(a)$ is the $k$-th differential of $P$ at $a$ (see [6], [11]).

\section{Dvoretzky-Rogers type theorems}

Two questions are treated in this section. The first question concerns a very useful result in the theory of summing linear operators, which happens to be a 
weak version of the celebrated Dvoretzky-Rogers Theorem and asserts that if $p \geq 1$ and $E$ is a Banach space, then

$E$ is finite dimensional $\Longleftrightarrow \mathscr{L}_{a s, p}(E ; E)=\mathscr{L}(E ; E)$.

For polynomials and multilinear mappings, Matos [9] proved that if $n>1$ and $p \geq 1$, then

$$
\begin{aligned}
E \text { is finite dimensional } & \Longleftrightarrow \mathscr{P}_{a s, p}^{e v}\left({ }^{n} E ; E\right)=\mathscr{P}\left({ }^{n} E ; E\right) \\
& \Longleftrightarrow \mathscr{L}_{a s, p}^{e v}\left({ }^{n} E ; E\right)=\mathscr{L}\left({ }^{n} E ; E\right) .
\end{aligned}
$$

On the other hand, for polynomials/multilinear mappings summing at the origin this result is not valid in general: for example, from [2, Theorems 2.2 and 2.5] we know that $\mathscr{P}_{a s, 1}\left({ }^{n} E ; E\right)=\mathscr{P}\left({ }^{n} E ; E\right)$ and $\mathscr{L}_{a s, 1}\left({ }^{n} E ; E\right)=\mathscr{L}\left({ }^{n} E ; E\right)$ for every $n \geq 2$ and every space $E$ of cotype 2 . The question is obvious: are there results of this type for polynomials and multilinear mappings summing at a point $a \neq 0$ ?

The second question arises from the well known fact that summability at the origin does not imply summability at a point $a \neq 0$ in general (see [9, Example 3.2]). Again the question is obvious: is it true that summability at some point $a \neq 0$ implies summability at the origin?

We solve these two questions in the affirmative. The multilinear and polynomial cases demand different reasonings.

\section{Multilinear case}

We start by showing some connections between $\mathscr{L}_{a s(p ; q)}^{(a)}$ and $\mathscr{L}_{a s(p ; q)}^{(b)}$ for $a \neq$ $b$. Some terminology is welcome. Given $T \in \mathscr{L}\left(E_{1}, \ldots, E_{n} ; F\right)$ and $a=$ $\left(a_{1}, \ldots, a_{n}\right) \in E_{1} \times \cdots \times E_{n}$, we denote by $T_{a_{1}}$ the $(n-1)$-linear mapping from $E_{2} \times \cdots \times E_{n}$ into $F$ given by

$$
T_{a_{1}}\left(x_{2}, \ldots, x_{n}\right)=T\left(a_{1}, x_{2}, \ldots, x_{n}\right) .
$$

Analogously we define the (n-1)-linear mappings $T_{a_{2}}, \ldots, T_{a_{n}}$, the $(n-2)$ linear mappings $T_{a_{1} a_{2}}=T\left(a_{1}, a_{2}, \cdot, \ldots, \cdot\right), \ldots, T_{a_{n-1} a_{n}}=T\left(\cdot, \ldots, \cdot, a_{n-1}\right.$, $\left.a_{n}\right)$ and the linear mappings $T_{a_{1}, \ldots, a_{n-1}}=T\left(a_{1}, \ldots, a_{n-1}, \cdot\right), \ldots, T_{a_{2}, \ldots, a_{n}}=$ $T\left(\cdot, a_{2}, \ldots, a_{n}\right)$.

Proposition 3.1. Let $a=\left(a_{1}, \ldots, a_{n}\right) \in E_{1} \times \cdots \times E_{n}$ and $T \in$ $\mathscr{L}_{a s\left(p ; q_{1}, \ldots, q_{n}\right)}^{(a)}\left(E_{1}, \ldots, E_{n} ; F\right)$. Then:

(a) $T_{a_{j_{1}}, \ldots, a_{j r}}$ is $\left(p ; q_{k_{1}}, \ldots q_{k_{s}}\right)$-summing at the origin whenever $\{1, \ldots, n\}=$ $\left\{j_{1}, \ldots, j_{r}\right\} \cup\left\{k_{1}, \ldots, k_{s}\right\}, k_{1} \leq \ldots \leq k_{s}$ and $\left\{j_{1}, \ldots, j_{r}\right\} \cap\left\{k_{1}, \ldots, k_{s}\right\}=\emptyset$.

(b) $T \in \mathscr{L}_{a s\left(p ; q_{1}, \ldots, q_{n}\right)}^{(b)}\left(E_{1}, \ldots, E_{n} ; F\right)$ for every $b \in\left\{\left(\lambda_{1} a_{1}, \ldots, \lambda_{n} a_{n}\right)\right.$; $\left.\lambda_{j} \in \mathrm{K}, j=1, \ldots, n\right\}$. 
So, the set of all points $b$ such that $T$ is $\left(p ; q_{1}, \ldots, p_{n}\right)$-summing at $b$ contains a linear subspace of $E_{1} \times \cdots \times E_{n}$. In particular, $T$ is $\left(p ; q_{1}, \ldots, q_{n}\right)$-summing at the origin.

Proof. (a) For the linear operator $T_{a_{1} \ldots a_{n-1}}$ it is enough to observe that $T_{a_{1} \ldots a_{n-1}}\left(x_{j}^{(n)}\right)=T\left(a_{1}+0, a_{2}+0, \ldots, a_{n-1}+0, a_{n}+x_{j}^{(n)}\right)-T\left(a_{1}, a_{2}, \ldots, a_{n}\right)$.

The cases of $T_{a_{1} \ldots a_{n-2} a_{n}}, \ldots, T_{a_{2} \ldots a_{n}}$ are analogous. For the bilinear mapping $T_{a_{1} \ldots a_{n-2}}$, observe that

$$
\begin{aligned}
T_{a_{1} \ldots a_{n-2}}\left(x_{j}^{(n-1)}, x_{j}^{(n)}\right) & \\
=[ & T\left(a_{1}+0, a_{2}+0, \ldots, a_{n-2}+0, a_{n-1}+x_{j}^{(n-1)}, a_{n}+x_{j}^{(n)}\right) \\
& \left.\quad-T\left(a_{1}, \ldots, a_{n}\right)\right]-T\left(a_{1}, a_{2}, \ldots, a_{n-1}, x_{j}^{(n)}\right) \\
\quad & \quad T\left(a_{1}, a_{2}, \ldots, a_{n-2}, x_{j}^{(n-1)}, a_{n}\right) \\
=[ & T\left(a_{1}+0, a_{2}+0, \ldots, a_{n-2}+0, a_{n-1}+x_{j}^{(n-1)}, a_{n}+x_{j}^{(n)}\right) \\
\quad & \left.\quad-T\left(a_{1}, \ldots, a_{n}\right)\right]-T_{a_{1}, \ldots, a_{n-1}}\left(x_{j}^{(n)}\right)-T_{a_{1}, \ldots, a_{n-2} a_{n}}\left(x_{j}^{(n-1)}\right) .
\end{aligned}
$$

$T$ is $\left(p ; q_{1}, \ldots, q_{n}\right)$-summing at $a$ by assumption and by the previous case we also know that $T_{a_{1}, \ldots, a_{n-1}}$ is $\left(p ; q_{n}\right)$-summing and $T_{a_{1}, \ldots, a_{n-2} a_{n}}$ is $\left(p ; q_{n-1}\right)$ summing, so it follows that $T_{a_{1} \ldots a_{n-2}}$ is $\left(p ; q_{n-1}, q_{n}\right)$-summing at the origin. The other cases of bilinear mappings are analogous. Proceeding in this line, the proof can be completed.

(b) Let $b=\left(\lambda_{1} a_{1}, \ldots, \lambda_{n} a_{n}\right)$. If $\lambda_{j} \neq 0$ for every $j$, it suffices to observe that

$$
\begin{aligned}
& \left(\sum_{j=1}^{\infty}\left\|T\left(\lambda_{1} a_{1}+x_{j}^{(1)}, \ldots, \lambda_{n} a_{n}+x_{j}^{(n)}\right)-T\left(\lambda_{1} a_{1}, \ldots, \lambda_{n} a_{n}\right)\right\|^{p}\right)^{\frac{1}{p}} \\
& =\left(\sum_{j=1}^{\infty}\left\|T\left(\lambda_{1} a_{1}+\frac{\lambda_{1}}{\lambda_{1}} x_{j}^{(1)}, \ldots, \lambda_{n} a_{n}+\frac{\lambda_{n}}{\lambda_{n}} x_{j}^{(n)}\right)-T\left(\lambda_{1} a_{1}, \ldots, \lambda_{n} a_{n}\right)\right\|^{p}\right)^{\frac{1}{p}} \\
& =\lambda_{1} \ldots \lambda_{n}\left(\sum_{j=1}^{\infty}\left\|T\left(a_{1}+\frac{1}{\lambda_{1}} x_{j}^{(1)}, \ldots, a_{n}+\frac{1}{\lambda_{n}} x_{j}^{(n)}\right)-T\left(a_{1}, \ldots, a_{n}\right)\right\|^{p}\right)^{\frac{1}{p}} .
\end{aligned}
$$

Now we use (a) to deal with the case in which $\lambda_{j}=0$ for some $j$. The case $n=3$ illustrates the reasoning: $T$ is $\left(p ; q_{1}, q_{2}, q_{3}\right)$-summing at $a=\left(a_{1}, a_{2}, a_{3}\right)$ by assumption, and from (a) we know that, at the origin, $T$ is $\left(p ; q_{1}, q_{2}, q_{3}\right)$ summing, $T_{a_{1}}$ is $\left(p ; q_{2}, q_{3}\right)$-summing, $T_{a_{2}}$ is $\left(p ; q_{1}, q_{3}\right)$-summing, $T_{a_{3}}$ is $\left(p ; q_{1}\right.$, 
$\left.q_{2}\right)$-summing, $T_{a_{1} a_{2}}$ is $\left(p ; q_{3}\right)$-summing, $T_{a_{1} a_{3}}$ is $\left(p ; q_{2}\right)$-summing and $T_{a_{2} a_{3}}$ is $\left(p ; q_{1}\right)$-summing.

- Case $\lambda_{1} \neq 0, \lambda_{2} \neq 0$ and $\lambda_{3}=0$ : follows from

$$
\begin{aligned}
T\left(\lambda_{1} a_{1}+x_{j}, \lambda_{2} a_{2}+y_{j}, z_{j}\right)-T\left(\lambda_{1} a_{1}, \lambda_{2} a_{2}, 0\right) \\
=\lambda_{1} \lambda_{2}\left[T\left(a_{1}+\frac{x_{j}}{\lambda_{1}}, a_{2}+\frac{y_{j}}{\lambda_{2}}, z_{j}\right)-T\left(a_{1}, a_{2}, 0\right)\right] \\
=\lambda_{1} \lambda_{2}\left[T\left(a_{1}, a_{2}, z_{j}\right)+T\left(\frac{x_{j}}{\lambda_{1}}, a_{2}, z_{j}\right)\right. \\
\left.\quad+T\left(a_{1}, \frac{y_{j}}{\lambda_{2}}, z_{j}\right)+T\left(\frac{x_{j}}{\lambda_{1}}, \frac{y_{j}}{\lambda_{2}}, z_{j}\right)\right] \\
\quad \lambda_{1} \lambda_{2}\left[T_{a_{1} a_{2}}\left(z_{j}\right)+T_{a_{2}}\left(\frac{x_{j}}{\lambda_{1}}, z_{j}\right)+T_{a_{1}}\left(\frac{y_{j}}{\lambda_{2}}, z_{j}\right)+T\left(\frac{x_{j}}{\lambda_{1}}, \frac{y_{j}}{\lambda_{2}}, z_{j}\right)\right] .
\end{aligned}
$$

- Cases $\lambda_{1}=0, \lambda_{2} \neq 0, \lambda_{3} \neq 0$ and $\lambda_{1} \neq 0, \lambda_{2}=0, \lambda_{3} \neq 0$ are analogous.

- Case $\lambda_{1} \neq 0, \lambda_{2}=\lambda_{3}=0$ : follows from

$$
\begin{aligned}
T\left(\lambda_{1} a_{1}+x_{j}, y_{j}, z_{j}\right)-T\left(\lambda_{1} a_{1}, 0,0\right) & =\lambda_{1}\left[T\left(a_{1}+\frac{x_{j}}{\lambda_{1}}, y_{j}, z_{j}\right)\right] \\
& =\lambda_{1}\left[T\left(a_{1}, y_{j}, z_{j}\right)+T\left(\frac{x_{j}}{\lambda_{1}}, y_{j}, z_{j}\right)\right] .
\end{aligned}
$$

- Cases $\lambda_{2} \neq 0, \lambda_{1}=\lambda_{3}=0$ and $\lambda_{3} \neq 0, \lambda_{2}=\lambda_{1}=0$ are analogous.

- Case $\lambda_{1}=\lambda_{2}=\lambda_{3}=0$ : we already know that $T$ is $\left(p ; q_{1}, q_{2}, q_{3}\right)$ summing at the origin.

The following result is a significant improvement of Matos' DvoretzkyRogers type theorem for multilinear mappings:

TheOREM 3.2. Let $E$ be a Banach space, $n \geq 2$ and $p \geq 1$. The following assertions are equivalent:

(a) E is infinite-dimensional.

(b) $\mathscr{L}_{a s, p}^{(a)}\left({ }^{n} E ; E\right) \neq \mathscr{L}\left({ }^{n} E ; E\right)$ for every $a=\left(a_{1}, \ldots, a_{n}\right) \in E^{n}$ with either $a_{i} \neq 0$ for every $i$ or $a_{i}=0$ for only one $i$.

(c) $\mathscr{L}_{a s, p}^{(a)}\left({ }^{n} E ; E\right) \neq \mathscr{L}\left({ }^{n} E ; E\right)$ for some $a=\left(a_{1}, \ldots, a_{n}\right) \in E^{n}$ with either $a_{i} \neq 0$ for every $i$ or $a_{i}=0$ for only one $i$.

Proof. Since (b) $\Rightarrow$ (c) is obvious and (c) $\Rightarrow$ (a) is a direct consequence of [9, Theorem 6.3], we just have to prove (a) $\Rightarrow$ (b): let $a=\left(a_{1}, \ldots, a_{n}\right) \in E^{n}$ with either $a_{i} \neq 0$ for every $i$ or $a_{i}=0$ for only one $i$. We can fix $k \in\{1, \ldots, n\}$ 
such that $a_{i} \neq 0$ for every $i \neq k$. For each $i \neq k$ choose $\varphi_{i} \in E^{\prime}$ so that $\varphi_{i}\left(a_{i}\right)=1$ and define $T \in \mathscr{L}\left({ }^{n} E ; E\right)$ by

$$
T\left(x_{1}, \ldots, x_{n}\right)=\varphi_{1}\left(x_{1}\right) \cdots \varphi_{k-1}\left(x_{k-1}\right) \varphi_{k+1}\left(x_{k+1}\right) \cdots \varphi_{n}\left(x_{n}\right) x_{k} .
$$

Since $T_{a_{1} \ldots a_{k-1} a_{k+1} \ldots a_{n}}(x)=T\left(a_{1}, \ldots, a_{k-1}, x, a_{k+1}, \ldots, a_{n}\right)=x$ for every $x \in E$, we have that $T_{a_{1} \ldots a_{k-1} a_{k+1} \ldots a_{n}}$ is not $p$-summing. From Proposition 3.1 it follows that $T$ is not $p$-summing at $a$.

From Proposition 3.1 we know that $\mathscr{L}_{a s, p}^{(a)}\left({ }^{n} E ; E\right)=\mathscr{L}\left({ }^{n} E ; E\right) \Longrightarrow$ $\mathscr{L}_{a s, p}\left({ }^{n} E ; E\right)=\mathscr{L}\left({ }^{n} E ; E\right)$. It is interesting to point out that Theorem 3.2 guarantees that much more holds in the bilinear case:

Corollary 3.3. Let $E$ be an infinite-dimensional Banach space, $a=$ $\left(a_{1}, \ldots, a_{n}\right) \in E^{n}, n \geq 2$ and $p \geq 1$. If $\mathscr{L}_{a s, p}^{(a)}\left({ }^{n} E ; E\right)=\mathscr{L}\left({ }^{n} E ; E\right)$, then $\operatorname{card}\left\{i: a_{i}=0\right\} \geq 2$. In particular, if $\mathscr{L}_{a s, p}^{(a)}\left({ }^{2} E ; E\right)=\mathscr{L}\left({ }^{2} E ; E\right)$ then $a$ is the origin.

REMARK 3.4. The condition $a_{i} \neq 0$ for every $i$ or $a_{i}=0$ for only one $i$ is essential in Theorem 3.2: for example, it is not difficult to check that $\mathscr{L}_{a s, 1}^{(a)}\left({ }^{n} \ell_{1} ; \ell_{1}\right)=\mathscr{L}\left({ }^{n} \ell_{1} ; \ell_{1}\right)$ for every $a=(x, 0,0, \ldots, 0)$ with $0 \neq x \in \ell_{1}$ and every $n \geq 3$.

\section{Polynomial case}

The theory of summing polynomials at a given point has some specific technical difficulties and deserves a precise examination. Despite the results we obtain for polynomials are analogous to the multilinear ones, the proofs of the multilinear results cannot be adapted to polynomials. For example, a polynomial version of Proposition 3.1 cannot be obtained following the lines of its proof. Such an adaptation would prove that if $P: E \longrightarrow F$ is $(p ; q)$-summing at $a \in E, a \neq 0$, then $P$ is $(p ; q)$-summing at every $\lambda a, \lambda \neq 0$. Indeed, this implication follows from

$$
\begin{aligned}
P\left(\lambda a+x_{j}\right)-P(\lambda a) & =P\left(\lambda a+\frac{\lambda}{\lambda} x_{j}\right)-P(\lambda a) \\
& =\lambda^{n}\left(P\left(a+\frac{1}{\lambda} x_{j}\right)-P(a)\right) .
\end{aligned}
$$

But we need more: we want to prove that if $P$ is $(p ; q)$-summing at $a \neq 0$, then $P$ is $(p ; q)$-summing at the origin. By $\check{P}$ we mean the unique symmetric continuous $n$-linear mapping associated to the $n$-homogeneous polynomial $P$. 
Proposition 3.5. Let $P \in \mathscr{P}\left({ }^{n} E ; F\right)$ and $a \in E$. $P$ is $(p ; q)$-summing at $a$ if and only if $\check{P}$ is $(p ; q, \ldots, q)$-summing at $(a, \ldots, a) \in E^{n}$.

Proof. Using the polarization formula, the case $a=0$ is immediate. We can suppose $a \neq 0$. Note that if $\check{P}$ is $(p ; q, \ldots, q)$-summing at $(a, \ldots, a)$ it is plain that $P$ is $(p ; q)$-summing at $a$. The proof of the other implication is divided in two cases: $n$ odd and $n$ even.

- First case: $n$ is odd. In this case the polarization formula is decisive:

$$
\begin{aligned}
n ! 2^{n}\left[\check{P}\left(a+x_{j}^{(1)}, \ldots, a+x_{j}^{(n)}\right)-\check{P}(a, \ldots, a)\right] \\
=\sum_{\varepsilon_{i}= \pm 1} \varepsilon_{1} \cdots \varepsilon_{n} P\left(\varepsilon_{1}\left(a+x_{j}^{(1)}\right)+\cdots+\varepsilon_{n}\left(a+x_{j}^{(n)}\right)\right) \\
\quad \quad-\sum_{\varepsilon_{i}= \pm 1} \varepsilon_{1} \cdots \varepsilon_{n} P\left(\varepsilon_{1} a+\cdots+\varepsilon_{n} a\right) \\
=\sum_{\varepsilon_{i}= \pm 1} \varepsilon_{1} \cdots \varepsilon_{n}\left[P\left(\left(\varepsilon_{1} a+\cdots+\varepsilon_{n} a\right)+\left(\varepsilon_{1} x_{j}^{(1)}+\cdots+\varepsilon_{n} x_{j}^{(n)}\right)\right)\right. \\
\left.\quad-P\left(\varepsilon_{1} a+\cdots+\varepsilon_{n} a\right)\right] .
\end{aligned}
$$

Since $n$ is odd, $\left(\varepsilon_{1}+\cdots+\varepsilon_{n}\right) \neq 0 . P$ is $(p ; q)$-summing at $a$ by assumption, so according to what we did above it follows that $P$ is $(p ; q)$-summing at each $\left(\varepsilon_{1} a+\cdots+\varepsilon_{n} a\right)$. Thus $(3.1)$ yields that $\check{P}$ is $(p ; q)$-summing at $(a, \ldots, a)$.

- Second case: $n$ is even. Choose $\varphi \in E^{\prime}$ so that $\varphi(a)=1$ and define $Q \in \mathscr{P}\left({ }^{n+1} E ; F\right)$ by $Q(x)=\varphi(x) P(x)$. Using that $P \in \mathscr{P}_{a s(p ; q)}^{(a)}\left({ }^{n} E ; F\right)$, it is easy to check that $Q$ is $(p ; q)$-summing at $a$. But $(n+1)$ is odd, so the previous case can be invoked in order to conclude that $\check{Q}$ is $(p ; q)$ summing at $(a, \ldots, a)$. Since $\check{Q}_{a}$ and $\varphi$ are $(p ; q)$-summing at the origin (the case of $\check{Q}_{a}$ follows from Proposition 3.1), from

$$
\check{Q}_{a}(x, \ldots, x)=\check{Q}(a, x, \ldots, x)=\frac{(n-1)}{n} \check{P}(a, x, \ldots, x) \varphi(x)+\frac{1}{n} P(x)
$$

we conclude that $P$ is $(p ; q)$-summing at the origin as well. Now, the polarization formula can be invoked as in (3.1) in order to conclude that $\stackrel{P}{P}$ is $(p ; q)$-summing at $(a, \ldots, a)$ and the proof is done.

Applying Proposition 3.1 once and Proposition 3.5 twice we have:

Corollary 3.6. Let $P \in \mathscr{P}\left({ }^{n} E ; F\right)$ be $(p ; q)$-summing at $a \in E$. Then $P$ is $(p ; q)$-summing at $\lambda$ a for every $\lambda \in \mathrm{K}$. In particular, $P$ is $(p ; q)$-summing at the origin.

Now we obtain the Dvoretzky-Rogers type theorem for polynomials summing at a point $a \neq 0$. 
Theorem 3.7. Let $E$ be a Banach space, $n \geq 2$ and $p \geq 1$. The following assertions are equivalent:

(a) $E$ is infinite-dimensional.

(b) $\mathscr{P}_{a s, p}^{(a)}\left({ }^{n} E ; E\right) \neq \mathscr{P}\left({ }^{n} E ; E\right)$ for every $a \in E, a \neq 0$.

(c) $\mathscr{P}_{a s, p}^{(a)}\left({ }^{n} E ; E\right) \neq \mathscr{P}\left({ }^{n} E ; E\right)$ for some $a \in E, a \neq 0$.

Proof. As in the proof of Theorem 3.2, we just have to prove (a) $\Rightarrow$ (b): let $a \in E, a \neq 0$. Choose $\varphi \in E^{\prime}$ so that $\varphi(a)=1$ and define $P \in \mathscr{P}\left({ }^{n} E ; E\right)$ by $P(x)=\varphi(x)^{n-1} x$. Assume that $P$ is $p$-summing at $a$. By Proposition 3.5 we have that $\breve{P}$ is $p$-summing at $(a, \ldots, a)$. Defining $P_{a} \in \mathscr{L}(E ; E)$ by $P_{a}(x)=\check{P}(a, \ldots, a, x)$, from

$$
P_{a}(x)=\check{P}(a+0, \ldots, a+0, a+x)-\check{P}(a, \ldots, a) \quad \text { for every } \quad x \in E,
$$

we conclude that $P_{a}$ is $p$-summing. From

$$
P_{a}(x)=\frac{(n-1)}{n} \varphi(x) a+\frac{1}{n} x \quad \text { for every } \quad x \in E,
$$

it follows that the identity operator on $E$ is $p$-summing. This contradiction completes the proof.

\section{Norms on spaces of everywhere summing polynomials}

In order to define a norm on the space $\mathscr{P}_{a s(p ; q)}^{e v}\left({ }^{n} E ; F\right)$ of everywhere $(p ; q)$-summing polynomials, Matos [9], in a clever argument, for each $P \in$ $\mathscr{P}_{a s(p ; q)}^{e v}\left({ }^{n} E ; F\right)$ considered the polynomial

$$
\Psi_{p ; q}(P): \ell_{q}^{u}(E) \longrightarrow \ell_{p}(F) ;\left(x_{j}\right)_{j=1}^{\infty} \longmapsto\left(P\left(x_{1}\right),\left(P\left(x_{1}+x_{j}\right)-P\left(x_{1}\right)\right)_{j=2}^{\infty}\right)
$$

and showed that the the correspondence $P \longrightarrow\left\|\Psi_{p ; q}(P)\right\|$ defines a norm on $\mathscr{P}_{a s(p ; q)}^{e v}\left({ }^{n} E ; F\right)$. We shall denote this norm by $\|P\|_{e v^{(1)}(p ; q)}$. Matos proved that this norm is complete and that $\left(\mathscr{P}_{a s(p ; q)}^{e v},\|\cdot\|_{e v^{(1)}(p ; q)}\right)$ is a global holomorphy type. Matos' argument was recently adapted to multilinear mappings in [3] (henceforth we whall write $\mathscr{L}_{a s(p ; q)}^{e v}$ instead of $\mathscr{L}_{a s(p ; q, \ldots, q)}^{e v}$ ): given $T \in$ $\mathscr{L}_{a s(p, q)}^{e v}\left(E_{1}, \ldots, E_{n} ; F\right)$, consider the multilinear mapping $\xi_{p ; q}(T): \ell_{q}^{u}\left(E_{1}\right) \times$ $\cdots \times \ell_{q}^{u}\left(E_{n}\right) \longrightarrow \ell_{p}(F)$ given by

$$
\begin{aligned}
&\left(\left(x_{j}^{(1)}\right)_{j=1}^{\infty}, \ldots,\left(x_{j}^{(n)}\right)_{j=1}^{\infty}\right) \longmapsto\left(T\left(x_{1}^{(1)}, \ldots, x_{1}^{(n)}\right),\right. \\
&\left.\left(T\left(x_{1}^{(1)}+x_{j}^{(1)}, \ldots, x_{1}^{(n)}+x_{j}^{(n)}\right)-T\left(x_{1}^{(1)}, \ldots, x_{1}^{(n)}\right)\right)_{j=2}^{\infty}\right) .
\end{aligned}
$$

In [3] it is proved that the correspondence $T \longrightarrow\left\|\xi_{p, q}(T)\right\|$ defines a complete norm on $\mathscr{L}_{a s(p ; q)}^{e v}\left({ }^{n} E ; F\right)$, which we shall denote by $\|T\|_{e v^{(1)}(p ; q)}$. So, in $\mathscr{P}_{a s(p ; q)}^{e v}$ 
another natural norm is defined by $\|P\|_{e v^{(I)}(p ; q)}:=\|\check{P}\|_{e v^{(1)}(p ; q)}$. In [3] it is shown that with this norm $\mathscr{P}_{a s(p ; q)}^{e v}$ is also a global holomorphy type.

We will see that these ideal norms on $\mathscr{P}_{a s(p ; q)}^{e v}$ and $\mathscr{L}_{a s(p ; q)}^{e v}$ are non-normalized in general and present quite serious difficulties concerning computations, even for very simple mappings. Our aim in this section is to introduce normalized ideal norms on $\mathscr{P}_{a s(p ; q)}^{e v}$ and $\mathscr{L}_{a s(p ; q)}^{e v}$ which happen to be equivalent to the original norms and make computations quite easier.

Next two theorems are adaptations of Matos' argument.

THEOREM 4.1. The following assertions are equivalent for $T \in \mathscr{L}\left(E_{1}, \ldots\right.$, $\left.E_{n} ; F\right)$ :

(a) $T \in \mathscr{L}_{a s(p ; q)}^{e v}\left(E_{1}, \ldots, E_{n} ; F\right)$.

(b) There exists $C$ such that

$$
\begin{aligned}
\left(\sum_{j=1}^{\infty} \| T\left(b_{1}\right.\right. & \left.\left.+x_{j}^{(1)}, \ldots, b_{n}+x_{j}^{(n)}\right)-T\left(b_{1}, \ldots, b_{n}\right) \|^{p}\right)^{\frac{1}{p}} \\
& \leq C\left(\left\|b_{1}\right\|+\left\|\left(x_{j}^{(1)}\right)_{j=1}^{\infty}\right\|_{w, q}\right) \ldots\left(\left\|b_{n}\right\|+\left\|\left(x_{j}^{(n)}\right)_{j=1}^{\infty}\right\|_{w, q}\right)
\end{aligned}
$$

for every $\left(b_{1}, \ldots, b_{n}\right) \in E_{1} \times \cdots \times E_{n}$ and $\left(x_{j}^{(k)}\right)_{j=1}^{\infty} \in \ell_{q}^{u}\left(E_{k}\right), k=1, \ldots, n$. Moreover, the infimum of all $C$ for which (b) holds defines a complete norm on $\mathscr{L}_{a s(p ; q)}^{e v}$ denoted by $\|\cdot\|_{e v^{(2)}(p ; q)}$.

Proof. Since (b) $\Rightarrow$ (a) is obvious we just have to prove (a) $\Rightarrow$ (b): define $G_{k}=E_{k} \times \ell_{q}^{u}\left(E_{k}\right), k=1, \ldots, n$, and consider the $n$-linear mapping $\Phi_{p ; q}(T)$ : $G_{1} \times \cdots \times G_{n} \longrightarrow \ell_{p}(F)$ given by

$$
\begin{aligned}
\left(\left(a_{1},\left(x_{j}^{(1)}\right)_{j=1}^{\infty}\right), \ldots\right. & \left.,\left(a_{n},\left(x_{j}^{(n)}\right)_{j=1}^{\infty}\right)\right) \\
& \longmapsto\left(T\left(a_{1}+x_{j}^{(1)}, \ldots, a_{n}+x_{j}^{(n)}\right)-T\left(a_{1}, \ldots, a_{n}\right)\right)_{j=1}^{\infty}
\end{aligned}
$$

Following the lines of the proofs of [3, Propositions 9.3 and 9.4] it can be proved that $\Phi_{p ; q}(T)$ is continuous and that the correspondence $T \longrightarrow\left\|\Phi_{p ; q}(T)\right\|:=$ $\|T\|_{e v^{(2)}(p ; q)}$ defines a complete norm on $\mathscr{L}_{a s(p ; q)}^{e v}\left(E_{1}, \ldots, E_{n} ; F\right)$.

THEOREM 4.2. The following assertions are equivalent for $P \in \mathscr{P}\left({ }^{n} E ; F\right)$ :

(a) $P \in \mathscr{P}_{a s(p ; q)}^{e v}\left({ }^{n} E ; F\right)$.

(b) There exists $C$ such that

$$
\left(\sum_{j=1}^{\infty}\left\|P\left(a+x_{j}\right)-P(a)\right\|^{p}\right)^{\frac{1}{p}} \leq C\left(\|a\|+\left\|\left(x_{j}\right)_{j=1}^{\infty}\right\|_{w, q}\right)^{n}
$$


for every $a \in E$ and $\left(x_{j}\right)_{j=1}^{\infty} \in \ell_{q}^{u}(E)$. Moreover, the infimum of all $C$ for which (b) holds defines a complete norm on $\mathscr{P}_{a s(p ; q)}^{e v}\left({ }^{n} E ; F\right)$ denoted by $\|\cdot\|_{e v^{(2)}(p ; q)}$.

Proof. Again we just have to prove (a) $\Rightarrow$ (b): define $G=E \times \ell_{q}^{u}(E)$ and consider the polynomial

$$
\eta_{p ; q}(P): G \longrightarrow \ell_{p}(F) ;\left(a,\left(x_{j}\right)_{j=1}^{\infty}\right) \longmapsto\left(P\left(a+x_{j}\right)-P(a)\right)_{j=1}^{\infty} .
$$

Following the lines of the proofs of [9, Theorem 7.2 and Proposition 7.4] it can be proved that $\eta_{p ; q}(P)$ is continuous and that the correspondence $P \longrightarrow$ $\left\|\eta_{p ; q}(P)\right\|:=\|P\|_{e v^{(2)}(p ; q)}$ defines a complete norm on $\mathscr{P}_{a s(p ; q)}^{e v}\left({ }^{n} E ; F\right)$.

We can also consider the norm on $\mathscr{P}_{a s(p, q)}^{e v}$ defined by $\|P\|_{e v^{(I I)}(p ; q)}:=$ $\|\check{P}\|_{e v^{(2)}(p ; q)}$. So we have four norms on $\mathscr{P}_{a s(p, q)}^{e v}$, namely $\|\cdot\|_{e v^{(1)}(p ; q)}$, $\|\cdot\|_{e v^{(2)}(p ; q)},\|\cdot\|_{e v^{(I)}(p ; q)}$ and $\|\cdot\|_{e v^{(I)}(p ; q)}$. We will show that: (i) these four norms are distinct in general but equivalent; (ii) the ideal $\left(\mathscr{P}_{a s(p, q)}^{e v},\|\cdot\|_{e v^{(2)}(p ; q)}\right)$ is normalized; (iii) the ideal $\left(\mathscr{P}_{a s(p, q)}^{e v},\|\cdot\|_{e v^{(1)}(p ; q)}\right)$ is non-normalized in general; (iv) the norm $\|\cdot\|_{e v^{(2)}(p ; q)}$ is easier for computations; (v) these four norms make $\mathscr{P}_{a s(p, q)}^{e v}$ a global holomorphy type. In our opinion these facts show that $\|\cdot\|_{e v^{(2)}(p ; q)}$ is the most convenient norm on $\mathscr{P}_{a s(p, q)}^{e v}$ and justify its introduction.

\section{Multilinear case}

Given $n \in \mathrm{N}$, by $A_{n}: \mathrm{K}^{n} \longrightarrow \mathrm{K}$ we mean the canonical $n$-linear mapping given by $A_{n}\left(x_{1}, \ldots, x_{n}\right)=x_{1} \cdots x_{n}$. According to the usual axiomatization, a Banach ideal of multilinear mappings $\left(\mathscr{M},\|\cdot\|_{\mathscr{M}}\right)$ must satisfy the condition $\left\|A_{n}\right\|_{M}=1$ for every $n$.

Proposition 4.3. Let $n \in \mathrm{N}$.

(a) $\left\|A_{n}\right\|_{e v^{(2)}(p ; q)}=1$ for every $p \geq q \geq 1$.

(b) $\left\|A_{n}\right\|_{e v^{(1)}(p ; 1)}=1$ for every $p \geq 1$.

(c) $\left\|A_{n}\right\|_{e v^{(1)}(p ; q)} \geq 2^{\frac{1}{q^{*}}}$, where $\frac{1}{q}+\frac{1}{q^{*}}=1$, for every $p \geq q>1$. In particular, $\left\|A_{n}\right\|_{e v^{(1)}(p ; q)}>1$ whenever $q>1$.

(d) $\lim _{n \rightarrow \infty}\left\|A_{n}\right\|_{e v^{(1)}(p ; q)}=\infty$ for every $p \geq q>1$.

Proof. By definition it is obvious that $\left\|A_{n}\right\|_{e v^{(1)}(p ; q)} \geq\left\|A_{n}\right\|_{a s(p ; q)}=1$ and $\left\|A_{n}\right\|_{e v^{(2)}(p ; q)} \geq\left\|A_{n}\right\|_{a s(p ; q)}=1$.

(a) We just have to prove that $\left\|A_{n}\right\|_{e v^{(2)}(p ; q)} \leq 1$. The case $n=3$ is illustrative: given $a_{1}, a_{2}, a_{3} \in \mathrm{K}$ and $\left(x_{j}^{1}\right),\left(x_{j}^{2}\right),\left(x_{j}^{3}\right) \in \ell_{q}=\ell_{q}^{u}(\mathrm{~K})$, since $p \geq q$ 
we have

$$
\begin{aligned}
&\left(\sum_{j=1}^{\infty}\left|A_{3}\left(a_{1}+x_{j}^{1}, a_{2}+x_{j}^{2}, a_{3}+x_{j}^{3}\right)-A_{3}\left(a_{1}, a_{2}, a_{3}\right)\right|^{p}\right)^{\frac{1}{p}} \\
&=\left(\sum_{j=1}^{\infty}\left|a_{1} a_{2} x_{j}^{3}+a_{1} a_{3} x_{j}^{2}+a_{1} x_{j}^{2} x_{j}^{3}+a_{2} a_{3} x_{j}^{1}+a_{2} x_{j}^{1} x_{j}^{3}+a_{3} x_{j}^{1} x_{j}^{2}+x_{j}^{1} x_{j}^{2} x_{j}^{3}\right|^{p}\right)^{\frac{1}{p}} \\
& \leq\left|a_{1} a_{2}\right|\left(\sum_{j=1}^{\infty}\left|x_{j}^{3}\right|^{q}\right)^{\frac{1}{q}}+\left|a_{1} a_{3}\right|\left(\sum_{j=1}^{\infty}\left|x_{j}^{2}\right|^{q}\right)^{\frac{1}{q}}+\left|a_{1}\right|\left(\sum_{j=1}^{\infty}\left|x_{j}^{2} x_{j}^{3}\right|^{q}\right)^{\frac{1}{q}}+\left|a_{2} a_{3}\right|\left(\sum_{j=1}^{\infty}\left|x_{j}^{1}\right|^{q}\right)^{\frac{1}{q}} \\
&+\left|a_{2}\right|\left(\sum_{j=1}^{\infty}\left|x_{j}^{1} x_{j}^{3}\right|^{q}\right)^{\frac{1}{q}}+\left|a_{3}\right|\left(\sum_{j=1}^{\infty}\left|x_{j}^{1} x_{j}^{2}\right|^{q}\right)^{\frac{1}{q}}+\left(\sum_{j=1}^{\infty}\left|x_{j}^{1} x_{j}^{2} x_{j}^{3}\right|^{q}\right)^{\frac{1}{q}} \\
& \leq\left|a_{1} a_{2}\right|\left(\sum_{j=1}^{\infty}\left|x_{j}^{3}\right|^{q}\right)^{\frac{1}{q}}+\left|a_{1} a_{3}\right|\left(\sum_{j=1}^{\infty}\left|x_{j}^{2}\right|^{q}\right)^{\frac{1}{q}}+\left|a_{1}\right|\left[\left(\sum_{j=1}^{\infty}\left|x_{j}^{2}\right|^{q}\right)\left(\sum_{j=1}^{\infty}\left|x_{j}^{3}\right|^{q}\right)\right]^{\frac{1}{q}} \\
&+\left|a_{2} a_{3}\right|\left(\sum_{j=1}^{\infty}\left|x_{j}^{1}\right|^{q}\right)^{\frac{1}{q}}+\left|a_{2}\right|\left[\left(\sum_{j=1}^{\infty}\left|x_{j}^{1}\right|^{q}\right)\left(\sum_{j=1}^{\infty}\left|x_{j}^{3}\right|^{q}\right)\right]^{\frac{1}{q}} \\
&+\left|a_{3}\right|\left[\left(\sum_{j=1}^{\infty}\left|x_{j}^{1}\right|^{q}\right)\left(\sum_{j=1}^{\infty}\left|x_{j}^{2}\right|^{q}\right)\right]^{\frac{1}{q}}+\left[\left(\sum_{j=1}^{\infty}\left|x_{j}^{1}\right|^{q}\right)\left(\sum_{j=1}^{\infty}\left|x_{j}^{2}\right|^{q}\right)\left(\sum_{j=1}^{\infty}\left|x_{j}^{3}\right|^{q}\right)\right]^{\frac{1}{q}} \\
&=\left(\left|a_{1}\right|+\left(\sum_{j=1}^{\infty}\left|x_{j}^{1}\right|^{q}\right)^{\frac{1}{q}}\right)\left(\left|a_{2}\right|+\left(\sum_{j=1}^{\infty}\left|x_{j}^{2}\right|^{q}\right)^{\frac{1}{q}}\right)\left(\left|a_{3}\right|+\left(\sum_{j=1}^{\infty}\left|x_{j}^{3}\right|^{q}\right)^{\frac{1}{q}}\right)-\left|a_{1} a_{2} a_{3}\right| \\
&=\left(\left|a_{1}\right|+\left\|\left(x_{j}^{1}\right)\right\|_{q}\right)\left(\left|a_{2}\right|+\left\|\left(x_{j}^{2}\right)\right\|_{w, q}\right)\left(\left|a_{2}\right|+\left\|\left(x_{j}^{2}\right)\right\|_{w, q}\right)\left(\left|a_{3}\right|+\left\|\left(x_{j}^{3}\right)\right\|_{q}\right) \\
&\left.\left(\| x_{j}^{3}\right) \|_{w, q}\right)
\end{aligned}
$$

proving that $\left\|A_{3}\right\|_{e v^{(2)}(p ; q)} \leq 1$.

(b) In essence, the same argument of (a). Use that $p \geq 1$ implies $\|\cdot\|_{p} \leq\|\cdot\|_{1}$ and in the case $q=1$, the last line of the above computation coincides with

$$
\left\|\left(a_{1},\left(x_{j}^{1}\right)\right)\right\|_{w, 1} \cdot\left\|\left(a_{2},\left(x_{j}^{2}\right)\right)\right\|_{w, 1} \cdot\left\|\left(a_{3},\left(x_{j}^{3}\right)\right)\right\|_{w, 1} .
$$

(c) We know that

$$
\begin{aligned}
& \left(\left|a_{1} \cdots a_{n}\right|^{p}+\sum_{j=1}^{\infty}\left|\left(a_{1}+x_{j}^{1}\right) \cdots\left(a_{n}+x_{j}^{n}\right)-a_{1} \cdots a_{n}\right|^{p}\right)^{\frac{1}{p}} \\
& \quad \leq\left\|A_{n}\right\|_{e v^{(1)}(p ; q)}\left(\left|a_{1}\right|^{q}+\sum_{j=1}^{\infty}\left|x_{j}^{1}\right|^{q}\right)^{\frac{1}{q}} \cdots\left(\left|a_{n}\right|^{q}+\sum_{j=1}^{\infty}\left|x_{j}^{n}\right|^{q}\right)^{\frac{1}{q}}
\end{aligned}
$$

for every $a_{k} \in \mathrm{K}$ and $\left(x_{j}^{k}\right)_{j=1}^{\infty} \in \ell_{q}, k=1, \ldots, n$. Choosing $a_{1}=\cdots=$ 
$a_{n-1}=0, a_{n}=1$ and $\left(x_{j}^{k}\right)_{j=1}^{\infty}=(1,0,0, \ldots)$ for $k=1, \ldots, n$, we have $2 \leq\left\|A_{n}\right\|_{e v^{(1)}(p ; q)} 2^{\frac{1}{q}}$, so $\left\|A_{n}\right\|_{e v^{(1)}(p ; q)} \geq 2^{1-\frac{1}{q}}=2^{\frac{1}{q^{*}}}$.

(d) Making $a_{1}=\cdots=a_{n}=1$ and $\left(x_{j}^{k}\right)_{j=1}^{\infty}=(1,0,0, \ldots)$ for $k=$ $1, \ldots, n$, in (4.2) we obtain

$$
\left(1+\left(2^{n}-1\right)^{p}\right)^{\frac{1}{p}} \leq\left\|A_{n}\right\|_{e v^{(1)}(p ; q)} 2^{\frac{n}{q}} .
$$

So,

$$
\left\|A_{n}\right\|_{e v^{(1)}(p ; q)} \geq \frac{\left(1+\left(2^{n}-1\right)^{p}\right)^{\frac{1}{p}}}{2^{\frac{n}{q}}} \longrightarrow \infty \quad \text { if } \quad n \longrightarrow \infty .
$$

Polynomial case

Given $n \in \mathrm{N}$, by $P_{n}: \mathrm{K} \longrightarrow \mathrm{K}$ we mean the canonical $n$-homogeneous polynomial given by $P_{n}(x)=x^{n}$. According to the usual axiomatization, a Banach ideal of homogeneous polynomials $\left(\mathscr{Q},\|\cdot\|_{\mathscr{Q}}\right)$ must satisfy the condition $\left\|P_{n}\right\|_{2}=1$ for every $n$.

Proposition 4.4. Let $n \in \mathrm{N}$.

(a) $\left\|P_{n}\right\|_{e v^{(2)}(p ; q)}=1$ for every $p \geq q \geq 1$.

(b) $\left\|P_{n}\right\|_{e v^{(1)}(p ; 1)}=1$ for every $p \geq 1$.

(c) $\lim _{n \rightarrow \infty}\left\|P_{n}\right\|_{e v^{(1)}(p ; q)}=\infty$ for every $p \geq q>1$.

Proof. By definition it is obvious that $\left\|P_{n}\right\|_{e v^{(1)}(p ; q)} \geq\left\|P_{n}\right\|_{a s(p ; q)}=1$ and $\left\|P_{n}\right\|_{e v^{(2)}(p ; q)} \geq\left\|P_{n}\right\|_{a s(p ; q)}=1$.

(a) We just have to prove that $\left\|P_{n}\right\|_{e v^{(2)}(p ; q)} \leq 1$. Given $a \in \mathrm{K}$ and $\left(x_{j}\right) \in \ell_{q}$, since $p \geq q$ we have

$$
\begin{aligned}
& \left(\sum_{j=1}^{\infty}\left|P_{n}\left(a+x_{j}\right)-P_{n}(a)\right|^{p}\right)^{\frac{1}{p}}=\left(\sum_{j=1}^{\infty}\left|\left(a+x_{j}\right)^{n}-a^{n}\right|^{p}\right)^{\frac{1}{p}} \\
& =\left(\sum_{j=1}^{\infty}\left|n a^{n-1} x_{j}+\left(\begin{array}{l}
n \\
2
\end{array}\right) a^{n-2} x_{j}^{2}+\cdots+\left(\begin{array}{l}
n \\
2
\end{array}\right) a^{2} x_{j}^{n-2}+n a x_{j}^{n-1}+x_{j}^{n}\right|^{p}\right)^{\frac{1}{p}} \\
& \leq n|a|^{n-1}\left(\sum_{j=1}^{\infty}\left|x_{j}\right|^{q}\right)^{\frac{1}{q}}+\left(\begin{array}{l}
n \\
2
\end{array}\right)|a|^{n-2}\left(\sum_{j=1}^{\infty}\left|x_{j}\right|^{2 q}\right)^{\frac{1}{q}}+ \\
& \cdots+n|a|\left(\sum_{j=1}^{\infty}\left|x_{j}\right|^{(n-1) q}\right)^{\frac{1}{q}}+\left(\sum_{j=1}^{\infty}\left|x_{j}\right|^{n q}\right)^{\frac{1}{q}}
\end{aligned}
$$




$$
\begin{gathered}
\leq n|a|^{n-1}\left(\sum_{j=1}^{\infty}\left|x_{j}\right|^{q}\right)^{\frac{1}{q}}+\left(\begin{array}{l}
n \\
2
\end{array}\right)|a|^{n-2}\left(\sum_{j=1}^{\infty}\left|x_{j}\right|^{q}\right)^{\frac{2}{q}}+ \\
\cdots+n|a|\left(\sum_{j=1}^{\infty}\left|x_{j}\right|^{q}\right)^{\frac{n-1}{q}}+\left(\sum_{j=1}^{\infty}\left|x_{j}\right|^{q}\right)^{\frac{n}{q}} \\
\leq\left(|a|+\left(\sum_{j=1}^{\infty}\left|x_{j}\right|^{q}\right)^{\frac{1}{q}}\right)^{n}=\left(|a|+\left\|\left(x_{j}\right)\right\|_{q}\right)^{n}=\left(|a|+\left\|\left(x_{j}\right)\right\|_{w, q}\right)^{n},
\end{gathered}
$$

proving that $\left\|P_{n}\right\|_{e v^{(2)}(p ; q)} \leq 1$.

(b) Essentially the same proof of (a) with $q=1$, using that $\left(|a|+\left\|\left(x_{j}\right)\right\|_{w, 1}\right)$ $=\left\|\left(a,\left(x_{j}\right)\right)\right\|_{w, 1}$.

(c) Repeating the multilinear argument, making $a=1$ and $\left(x_{j}\right)_{j=1}^{\infty}=$ $(1,0,0, \ldots)$ we obtain

$$
\left\|P_{n}\right\|_{e v^{(1)}(p ; q)} \geq \frac{\left(1+\left(2^{n}-1\right)^{p}\right)^{\frac{1}{p}}}{2^{\frac{n}{q}}} \longrightarrow \infty \quad \text { if } \quad n \longrightarrow \infty .
$$

Next examples show that the four norms on $\mathscr{P}_{a s(p ; q)}^{e v}$ are different in general.

EXAMPLE 4.5. From Propositions 4.3 and 4.4 we already know that, in most cases,

$$
\|\cdot\|_{e v^{(1)}(p ; q)} \neq\|\cdot\|_{e v^{(2)}(p ; q)}
$$

for multilinear mappings and for polynomials. In particular, for appropriate $n$, $p$ and $q$, since $A_{n}=\left(P_{n}\right)^{\vee}$ we have

$$
\left\|P_{n}\right\|_{e v^{(1)}(p ; q)} \neq\left\|P_{n}\right\|_{e v^{(2)}(p ; q)}
$$

and

$$
\left\|P_{n}\right\|_{e v^{(I)}(p ; q)}=\left\|A_{n}\right\|_{e v^{(1)}(p ; q)} \neq\left\|A_{n}\right\|_{e v^{(2)}(p ; q)}=\left\|P_{n}\right\|_{e v^{(I)}(p ; q)} .
$$

EXAMPLE 4.6. Let us see that, for polynomials, $\|\cdot\|_{e v^{(2)}(p ; q)} \neq\|\cdot\|_{e v^{(I)}(p ; q)}$ in general. Let $Q_{2}$ be the 2nd Nachbin polynomial, that is

$$
\left.Q_{2}:\left(\mathrm{C}^{2},\|\cdot\|_{\ell_{1}}\right) \longrightarrow \mathrm{C}: Q_{n}(x, y)\right)=x y .
$$

So, $\left(Q_{2}\right)^{\vee}: \mathrm{C}^{2} \times \mathrm{C}^{2} \longrightarrow \mathrm{C}$ is given by $\left.\left(Q_{2}\right)^{\vee}\left(x_{1}, y_{1}\right),\left(x_{2}, y_{2}\right)\right)=\frac{x_{1} y_{2}+x_{2} y_{1}}{2}$. We shall prove that

$$
\left\|Q_{2}\right\|_{e v^{(2)}(1 ; 1)}=\frac{1}{4}<\frac{1}{2}=\left\|\left(Q_{2}\right)^{\vee}\right\|_{e v^{(2)}(1 ; 1)}=\left\|Q_{2}\right\|_{e v^{(I I)}(1 ; 1)} .
$$


Given $a=\left(a_{1}, a_{2}\right), b=\left(b_{1}, b_{2}\right) \in \mathrm{C}^{2}$ and $\left(x_{j}\right)=\left(\left(x_{j}^{1}, x_{j}^{2}\right)\right),\left(y_{j}\right)=$ $\left(\left(y_{j}^{1}, y_{j}^{2}\right)\right) \in \ell_{1}\left(\mathrm{C}^{2}\right)=\ell_{1}^{u}\left(\mathrm{C}^{2}\right)$,

$$
\begin{aligned}
\sum_{j=1}^{\infty} \mid & \left(Q_{2}\right)^{\vee}\left(a+x_{j}, b+y_{j}\right)-\left(Q_{2}\right)^{\vee}(a, b) \mid \\
= & \sum_{j=1}^{\infty}\left|\frac{\left(a_{1}+x_{j}^{1}\right)\left(b_{2}+y_{j}^{2}\right)+\left(a_{2}+x_{j}^{2}\right)\left(b_{1}+y_{j}^{1}\right)}{2}-\frac{\left(a_{1} b_{2}+a_{2} b_{1}\right)}{2}\right| \\
= & \frac{1}{2} \sum_{j=1}^{\infty}\left|a_{1} y_{j}^{2}+b_{2} x_{j}^{1}+a_{2} y_{j}^{1}+b_{1} x_{j}^{2}+x_{j}^{1} y_{j}^{2}+x_{j}^{2} y_{j}^{1}\right| \\
\leq & \frac{1}{2}\left(\sum_{j=1}^{\infty}\left|a_{1} y_{j}^{2}\right|+\sum_{j=1}^{\infty}\left|b_{2} x_{j}^{1}\right|+\sum_{j=1}^{\infty}\left|a_{2} y_{j}^{1}\right|\right. \\
& \left.+\sum_{j=1}^{\infty}\left|b_{1} x_{j}^{2}\right|+\sum_{j=1}^{\infty}\left|x_{j}^{1} y_{j}^{2}\right|+\sum_{j=1}^{\infty}\left|x_{j}^{2} y_{j}^{1}\right|\right) \\
\leq & \frac{1}{2}\left[\left|a_{1}\right| \sum_{j=1}^{\infty}\left|y_{j}^{2}\right|+\left|b_{2}\right| \sum_{j=1}^{\infty}\left|x_{j}^{1}\right|+\left|a_{2}\right| \sum_{j=1}^{\infty}\left|y_{j}^{1}\right|+\left|b_{1}\right| \sum_{j=1}^{\infty}\left|x_{j}^{2}\right|\right. \\
& \left.+\left(\sum_{j=1}^{\infty}\left|x_{j}^{1}\right|\right)\left(\sum_{j=1}^{\infty}\left|y_{j}^{2}\right|\right)+\left(\sum_{j=1}^{\infty}\left|x_{j}^{2}\right|\right)\left(\sum_{j=1}^{\infty}\left|y_{j}^{1}\right|\right)\right] \\
\leq & \frac{1}{2}\left(\left|a_{1}\right|+\left|a_{2}\right|+\sum_{j=1}^{\infty}\left|x_{j}^{1}\right|+\sum_{j=1}^{\infty}\left|x_{j}^{2}\right|\right)\left(\left|b_{1}\right|+\left|b_{2}\right|+\sum_{j=1}^{\infty}\left|y_{j}^{1}\right|+\sum_{j=1}^{\infty}\left|y_{j}^{2}\right|\right) \\
= & \frac{1}{2}\left(\|a\|+\sum_{j=1}^{\infty}\left\|x_{j}\right\|\right)\left(\|b\|+\sum_{j=1}^{\infty}\left\|y_{j}\right\|\right) \\
= & \frac{1}{2}\left(\|a\|+\left\|\left(x_{j}\right)\right\|_{1}\right)\left(\|b\|+\left\|\left(y_{j}\right)_{1}\right\|\right),
\end{aligned}
$$

proving that $\left\|\left(Q_{2}\right)^{\vee}\right\|_{e v^{(2)}(1 ; 1)} \leq \frac{1}{2}$. Making

$$
a=(0,0), \quad b=(1,0), \quad\left(x_{j}\right)=((0,1),(0,0),(0,0), \ldots)
$$

and

$$
\left(y_{j}\right)=((0,0),(0,0),(0,0), \ldots),
$$

we obtain $\left\|\left(Q_{2}\right)^{\vee}\right\|_{e v^{(2)}(1 ; 1)} \geq \frac{1}{2}$. So $\left\|\left(Q_{2}\right)^{\vee}\right\|_{e v^{(2)}(1 ; 1)}=\frac{1}{2}$. 
Let $(a, b) \in \mathrm{C}^{2}$ and $\left(\left(x_{j}, y_{j}\right)\right) \in \ell_{1}\left(\mathrm{C}^{2}\right)=\ell_{1}^{u}\left(\mathrm{C}^{2}\right)$.

$$
\begin{aligned}
0 \leq & \left(|a|-|b|+\sum_{j=1}^{\infty}\left|x_{j}\right|-\sum_{j=1}^{\infty}\left|y_{j}\right|\right)^{2} \\
= & |a|^{2}+|b|^{2}-2|a b|+2|a| \sum_{j=1}^{\infty}\left|x_{j}\right|-2|a| \sum_{j=1}^{\infty}\left|y_{j}\right|-2|b| \sum_{j=1}^{\infty}\left|x_{j}\right| \\
& +2|b| \sum_{j=1}^{\infty}\left|y_{j}\right|-2\left(\sum_{j=1}^{\infty}\left|x_{j}\right|\right)\left(\sum_{j=1}^{\infty}\left|y_{j}\right|\right)+\left(\sum_{j=1}^{\infty}\left|x_{j}\right|\right)^{2}+\left(\sum_{j=1}^{\infty}\left|y_{j}\right|\right)^{2} .
\end{aligned}
$$

Adding $4|a| \sum_{j}\left|y_{j}\right|+4|b| \sum_{j}\left|x_{j}\right|+4\left(\sum_{j}\left|x_{j}\right|\right)\left(\sum_{j}\left|y_{j}\right|\right)$ in both sides, it follows that

$$
\begin{aligned}
& 4\left(\sum_{j=1}^{\infty}\left|Q_{2}\left((a, b)+\left(x_{j}, y_{j}\right)\right)-Q_{2}((a, b))\right|\right) \\
& =4\left(\sum_{j=1}^{\infty}\left|a y_{j}+b x_{j}+x_{j} y_{j}\right|\right) \\
& \leq 4\left(|a| \sum_{j=1}^{\infty}\left|y_{j}\right|+|b| \sum_{j=1}^{\infty}\left|x_{j}\right|+\sum_{j=1}^{\infty}\left|x_{j} y_{j}\right|\right) \\
& \leq 4\left(|a| \sum_{j=1}^{\infty}\left|y_{j}\right|+|b| \sum_{j=1}^{\infty}\left|x_{j}\right|+\left(\sum_{j=1}^{\infty}\left|x_{j}\right|\right)\left(\sum_{j=1}^{\infty}\left|y_{j}\right|\right)\right) \\
& \leq|a|^{2}+|b|^{2}-2|a b|+2|a| \sum_{j=1}^{\infty}\left|x_{j}\right|+2|a| \sum_{j=1}^{\infty}\left|y_{j}\right|+2|b| \sum_{j=1}^{\infty}\left|x_{j}\right| \\
& +2|b| \sum_{j=1}^{\infty}\left|y_{j}\right|+2\left(\sum_{j=1}^{\infty}\left|x_{j}\right|\right)\left(\sum_{j=1}^{\infty}\left|y_{j}\right|\right)+\left(\sum_{j=1}^{\infty}\left|x_{j}\right|\right)^{2}+\left(\sum_{j=1}^{\infty}\left|y_{j}\right|\right)^{2} \\
& \leq|a|^{2}+|b|^{2}+2|a b|+2|a| \sum_{j=1}^{\infty}\left|x_{j}\right|+2|a| \sum_{j=1}^{\infty}\left|y_{j}\right|+2|b| \sum_{j=1}^{\infty}\left|x_{j}\right| \\
& +2|b| \sum_{j=1}^{\infty}\left|y_{j}\right|+2\left(\sum_{j=1}^{\infty}\left|x_{j}\right|\right)\left(\sum_{j=1}^{\infty}\left|y_{j}\right|\right)+\left(\sum_{j=1}^{\infty}\left|x_{j}\right|\right)^{2}+\left(\sum_{j=1}^{\infty}\left|y_{j}\right|\right)^{2} \\
& =\left(|a|+|b|+\sum_{j=1}^{\infty}\left|x_{j}\right|+\sum_{j=1}^{\infty}\left|y_{j}\right|\right)^{2}=\left(\|(a, b)\|+\left\|\left(\left(x_{j}, y_{j}\right)\right)\right\|_{1}\right)^{2} \text {, }
\end{aligned}
$$


proving that $\left\|Q_{2}\right\|_{e v^{(2)}(1 ; 1)} \leq \frac{1}{4}$. Making $(a, b)=(1,0),\left(x_{j}\right)=(0,0, \ldots)$ and $\left(y_{j}\right)=(1,0,0, \ldots)$, we obtain $\left\|Q_{2}\right\|_{e v^{(2)}(1 ; 1)} \geq \frac{1}{4}$. So $\left\|Q_{2}\right\|_{e v^{(2)}(1 ; 1)}=\frac{1}{4}$.

Once we know that the four norms on $\mathscr{P}_{a s(p ; q)}^{e v}$ are different in general, we would like to prove that they are equivalent. There is no hope for them to be uniformly equivalent on $n$, because from Propositions 4.3(d) and 4.4(c) we know that, for $q>1$, there is neither a constant $C$ such that

$$
\left\|P_{n}\right\|_{e v^{(1)}(p ; q)} \leq C\left\|P_{n}\right\|_{e v^{(2)}(p ; q)} \quad \text { for every } n,
$$

nor a constant $C$ such that

$$
\left\|P_{n}\right\|_{e v^{(I)}(p ; q)} \leq C\left\|P_{n}\right\|_{e v^{(I I)}(p ; q)} \quad \text { for every } n .
$$

Proposition 4.7. For every natural $n$, real numbers $1 \leq q \leq p$, Banach spaces $E$ and $F$ and $P \in \mathscr{P}_{a s(p ; q)}^{e v}\left({ }^{n} E ; F\right)$,

$$
\|P\|_{e v^{(2)}(p ; q)} \leq\|P\|_{e v^{(1)}(p ; q)}, \quad\|P\|_{e v^{(2)}(p ; q)} \leq\|P\|_{e v^{(I)}(p ; q)} \leq e^{n}\|P\|_{e v^{(2)}(p ; q)}
$$

and

$$
\|P\|_{e v^{(1)}(p ; q)} \leq\|P\|_{e v^{(I)}(p ; q)} \leq e^{n}\|P\|_{e v^{(1)}(p ; q)} .
$$

Proof. Given $P \in \mathscr{P}_{a s(p ; q)}^{e v}\left({ }^{n} E ; F\right), a \in E$ and $\left(x_{j}\right) \in \ell_{q}^{u}(E)$, from

$$
\begin{aligned}
\left(\sum_{j=1}^{\infty}\right. & \left.\left\|P\left(a+x_{j}\right)-P(a)\right\|^{p}\right)^{\frac{1}{p}} \\
& \leq\left(\|P(a)\|^{p}+\sum_{j=1}^{\infty}\left\|P\left(a+x_{j}\right)-P(a)\right\|^{p}\right)^{\frac{1}{p}} \\
& \leq\|P\|_{e v^{(1)}(p ; q)} \sup _{\|\varphi\| \leq 1}\left(|\varphi(a)|^{q}+\sum_{j=1}^{\infty}\left|\varphi\left(x_{j}\right)\right|^{q}\right)^{\frac{n}{q}} \\
& \leq\|P\|_{e v^{(1)}(p ; q)}\left(\sup _{\|\varphi\| \leq 1}|\varphi(a)|^{q}+\sup _{\|\varphi\| \leq 1} \sum_{j=1}^{\infty}\left|\varphi\left(x_{j}\right)\right|^{q}\right)^{\frac{n}{q}} \\
& =\|P\|_{e v^{(1)}(p ; q)}\left(\|a\|^{q}+\left\|\left(x_{j}\right)\right\|_{w, q}^{q}\right)^{\frac{n}{q}} \\
& \leq\|P\|_{e v^{(1)}(p ; q)}\left(\|a\|+\left\|\left(x_{j}\right)\right\|_{w, q}\right)^{n},
\end{aligned}
$$

we conclude that $\|P\|_{e v^{(2)}(p ; q)} \leq\|P\|_{e v^{(1)}(p ; q)}$.

For every $P \in \mathscr{P}_{a s(p ; q)}^{e v}\left({ }^{n} E ; F\right)$ we know that

$$
\check{P} \in \mathscr{L}_{a s(p ; q)}^{e v}\left({ }^{n} E ; F\right), \quad\|P\|_{e v^{(2)}(p ; q)}=\left\|\eta_{p ; q}(P)\right\|
$$


and

$$
\|P\|_{e v^{(I I)}(p ; q)}=\|\check{P}\|_{e v^{(2)}(p ; q)}=\left\|\Phi_{p ; q}(\check{P})\right\|=\left\|\left(\eta_{p ; q}(P)\right)^{\vee}\right\|,
$$

because $\Phi_{p ; q}(\check{P})$ is symmetric and $\left(\Phi_{p ; q}(\check{P})\right)^{\wedge}=\eta_{p ; q}(P)$. From the classical estimates

$$
\left\|\eta_{p ; q}(P)\right\| \leq\left\|\left(\eta_{p ; q}(P)\right)^{\vee}\right\| \leq e^{n}\left\|\eta_{p ; q}(P)\right\|
$$

we obtain

$$
\|P\|_{e v^{(2)}(p ; q)} \leq\|P\|_{e v^{(I)}(p ; q)} \leq e^{n}\|P\|_{e v^{(2)}(p ; q)} .
$$

The remaining inequalities are analogous.

Corollary 4.8. Given $n \in \mathrm{N}, 1 \leq q \leq p$, Banach spaces $E$ and $F$, the norms $\|\cdot\|_{e v^{(1)}(p ; q)},\|\cdot\|_{e v^{(2)}(p ; q)},\|\cdot\|_{e v^{(I)}(p ; q)}$ and $\|\cdot\|_{e v^{(I)}(p ; q)}$ are equivalent on $\mathscr{P}_{\text {as }(p ; q)}\left({ }^{n} E ; F\right)$.

Proof. Just combine the Open Mapping Theorem with the inequalities of Proposition 4.7.

Proposition 4.9. For $\mathrm{K}=\mathrm{C}$, given $1 \leq q \leq p$, $\mathscr{P}_{a s(p ; q)}^{e v}$ is a global holomorphy type with either $\|\cdot\|_{e v^{(1)}(p ; q)},\|\cdot\|_{e v^{(2)}(p ; q)},\|\cdot\|_{e v^{(I)}(p ; q)}$ or $\|\cdot\|_{e v^{(I)}(p ; q)}$.

Proof. From [9, Proposition 7.8], $\left(\mathscr{P}_{a s(p ; q)}^{e v},\|\cdot\|_{e v^{(1)}(p ; q)}\right)$ is a global holomorphy type (with constant $2 e$ ) and an adaptation of [9, Proposition 7.8] provides that $\left(\mathscr{P}_{a s(p ; q)}^{e v},\|\cdot\|_{e v^{(2)}(p ; q)}\right)$ is a global holomorphy type. Combining these facts with the inequalities we proved in Proposition 4.7, we obtain that the other two norms also generate global holomorphy types.

\section{REFERENCES}

1. Alencar, R., and Matos, M. C., Some classes of multilinear mappings between Banach spaces, Publicaciones Departamento Análisis Matematico, Universidad Complutense Madrid, Section 1, Number 12 (1989).

2. Botelho, G., Cotype and absolutely summing multilinear mappings and homogeneous polynomials, Proc. Roy. Irish Acad. Sect. A 97 (1997), 145-153.

3. Botelho, G., Braunss, H-A., Junek, H., and Pellegrino, D., Holomorphy types and ideals of multilinear mappings, Studia Math. 177 (2006), 43-65.

4. Diestel, J., Jarchow, H., and Tonge, A., Absolutely Summing Operators, Cambridge Stud. Adv. Math. 43 (1995).

5. Dimant, V., Strongly p-summing multilinear mappings, J. Math. Anal. Appl. 278 (2003), $182-193$.

6. Dineen, S., Complex Analysis on Infinite Dimensional Spaces, Springer-Verlag, London, 1999.

7. Çaliskan, E., and Pellegrino, D., On multilinear extensions of absolutely summing operators, Rocky Mountain J. Math, to appear.

8. Matos, M. C., Absolutely summing holomorphic mappings, An. Acad. Brasil. Ciênc. 68 (1996), $1-13$. 
9. Matos, M. C., Nonlinear absolutely summing mappings, Math. Nachr. 258 (2003), 71-89.

10. Matos, M. C., Fully absolutely summing mappings and Hilbert Schmidt operators, Collect. Math. 54 (2003), 111-136.

11. Nachbin, L., Topology on Spaces of Holomorphic Mappings, Springer-Verlag, New York, 1969.

12. Pellegrino, D., Aplicações entre espaços de Banach relacionadas à convergência de séries, Doctoral Thesis, UNICAMP, 2002.

13. Pellegrino, D., Almost summing mappings, Arch. Math. 82 (2004), 68-80.

14. Pellegrino, D., Cotype and nonlinear absolutely summing mappings, Math. Proc. R. Ir. Acad. 105(A) (2005), 75-91.

15. Pérez-García, D., and Villanueva, I., Multiple summing operators on Banach spaces, J. Math. Anal. Appl. 285 (2003), 86-96.

16. Pietsch, A., Ideals of multilinear functionals (designs of a theory), Proceedings of the Second International Conference on Operator Algebras, Ideals and their Applications in Theoretical Physics, 185-199. Leipzig. Teubner-Texte, 1983.

FACULDADE DE MATEMÁTICA

UNIVERSIDADE FEDERAL DE UBERLÂNDIA

38.400-902-UBERLÂNDIA

BRAZIL

E-mail: botelho@ufu.br

DEPARTAMENTO DE MATEMÁTICA

UNIVERSIDADE FEDERAL DA PARAÍBA

58.051-900-JOÃO PESSOA

BRAZIL

E-mail: dmpellegrino@gmail.com
DEPARTAMENTO DE MATEMÁTICA E ESTATÍSTICA UNIVERSIDADE FEDERAL DE CAMPINA GRANDE 58.109-970-CAMPINA GRANDE

BRAZIL

E-mail: jaime@dme.ufcg.edu.br, diogodme@gmail.com 